\title{
Flow Past a Grooved Circular Cylinder
}

\author{
Gamal H. Moustafa and Alam El-din A. M. \\ Mechanical Power Engineering Dept. \\ Faculty of Engineering \\ Menoufia University, Shiben El-Kom \\ EGYPT
}

\begin{abstract}
An attempt is made to clearly distinguish the effect of a groove shape on the aerodynamic characteristics of a circular cylinder. The grooves are used as a passive control for the boundary layer separation. Grooves of different cross section shapes are made on the cylinder surface to investigate the effect of a regular surface configuration and to elucidate the flow structure in the wake region where the effect of grooves is existed. The results show that the grooves greatly affect the location of the separation point in which a shift of the flow regime to lower Reynolds number is found. The subcritical, critical and supercritical regimes can easily identified from flow measurements. It is found that the critical Reynolds number for tested grooved cylinders is $5 \times 10^{4}$ which are below the subcritical Reynolds number of a smooth cylinder. The critical Reynolds number for a smooth cylinder is $3 \times 10^{5}$. The main features of the boundary layer development are quit the same as that of the smooth cylinder. The boundary layer thickness and shape factor are large for grooved cylinders compared to that of the smooth cylinders and these results are in good agreement with previous available data.
\end{abstract}

Manuscript received from Dr: Gamal $H$. Moustafa at: 21/12/1997, accepted at: 9/5/1998, Enginecring research bulletin, Vol. 21, No. 2, 1998, Menoufiya University, Faculty of Engineering, Shcbin El- Kom, Egypt, ISSN. 1110-1180. 


\section{Introduction}

Flow around a circular cylinder is very important and has received the attention of aerodynamicistics. The applications of such a flow exist in many engineering fields. Flow around structures in the ocean and that in heat exchangers are typical examples of such applications. In these cases, the flow field is mostly affected by the surface characteristics of the cylindrical body.

It is well known that as a fluid flows across a cylinder it encounters a hydraulic resistance which is called drag. The total drag of the cylinder is equal to the sum of the viscous drag and the pressure drag. The viscous drag is mainly due to the frictional forces developed in the boundary layer on the frontal part of the cylinder. Although, these frictional forces only constitute a few percent of the total drag, they influence the flow around the cylinder by affecting the boundary layer development.

The aim of most previous researches is to reduce the drag. Hence, many techniques have been developed to reduce the fluid forces and to suppress vortex shedding over a circular cylinder. Passive controls are well known as methods for controlling and preventing the boundary layer separation. Artificial transition of the surface boundary layer by a tripwire and its effect on the drag coefficient was used by Schlichting(1979) and earlier by Naumann and Quadflieg (1972). The effects of surface roughness on the flow field was discussed by Achenbach (1971) and Kawamura and Kuwahara (1984). The surface roughness not only promoted earlier boundary layer transition but also significantly affected the subsequent flow development at higher Reynolds number, resulting in a shift of the flow regime to lower Reynolds numbers.

A large number of studies concerned with a flow around a grooved circular cylinder have been recorded. Attention has focused on the formation of the wake, the frequency of vortex shedding, and calculations of forces acting on the cylinder. The V-shape grooves was the common for most of the studies, Ko, et al. (1987) and Leung and Ko (1987). The circular arc groove on the surface of a circular cylinder as that exists on golf bolls was shown to have an effect on reducing drag on the cylinder, Bearman and Harvey (1976). In general, grooves have been used as a passive control for reducing the body drag. Experimental study made by Quass, et al. (1981) proved that longitudinal grooves on a bluff body reduced the associated drag by about 40\%. Also, Gregory and Farid (1990) studied the effect of surface grooves on the base pressure for a blunt trailing edge airfoil. The results show that the surface grooves affect the base pressure which changes the drag and lift coefficient. 
The aim of the present investigation is to study the effect of the groove shape on the flow field around a cylinder. Thus, to examine the flow field, the presence of vortices, factors governing their generation and its effect on the wake flow. Also, to investigate the effect of regular surface configuration on the flow field and boundary layer separation. The results presented herein may be useful for reducing the drag on two dimensional blunt cylinders, such as tall chimneys or bridge girders.

\section{Experimental Apparatus and Procedures}

Experiments were carried out in a low speed open type wind tunnel, Fig. 1. The test section was made of prespex with dimensions of height $=305 \mathrm{~mm}$, width $=305 \mathrm{~mm}$ and length $=610 \mathrm{~mm}$. The top of the test section was arranged to support a three dimensional traversing mechanism. This mechanism was used to traverse the probe in the longitudinal and lateral directions. An access port of $102 \mathrm{~mm}$ diameter was provided in the front side wall where the tested cylinder was inserted and a pin is engaged in a hole in the far side wall of the test section. The port was closed during the operation by a flanged Plexiglas play. The tunnel was designed to permit a free stream velocity up to $60 \mathrm{~m} / \mathrm{s}$. Thus, the motor speed was regulated by a transistor inverter type variable speed controller.

The test model was a circular cylinder of $50 \mathrm{~mm}$ diameter and $300 \mathrm{~mm}$ length. Four models of the same dimensions were tested as given in Fig. 2. The first model was a circular cylinder without grooves which will be known, hereafter as the smooth cylinder. Surface grooves of different cross sectional shapes were made for the other three models. The grooves were cut circumferentially with an interval of $\theta=36^{\circ}$, starting from the stagnation point. The depth of the grooves was $3 \mathrm{~mm}$, for all the test models. For cylinders $\mathrm{Cy} 1$ and $\mathrm{Cy} 2$, the grooves width was $3 \mathrm{~mm}$. The $\mathrm{V}$ - grooves were made with an angle of $19^{\circ}$. During the experiments, the cylinder were mounted horizontally with their axes perpendicular to the flow at a position half way between the roof and floor of the test section. The circular cylinder in a uniform shear flow and the coordinate system in the test section are illustrated in Fig. 3.

Measurements of pressures along and behind the cylinder were made at different initial flow velocities. The Reynolds number based on the cylinder diameter was ranged from $3.3 \times 10^{4}$ to $1.71 \times 10^{5}$. Taps of $0.5 \mathrm{~mm}$ inner diameter were made along the cylinder diameter in a midspan to measure the static pressure distribution. The first tap was positioned at the stagnation point. For grooved cylinders the taps were made at the bottom of the grooves. The base pressure was measured through a tap made at the base of the cylinder, Fig. 2. Measurements of the pressure behind the cylinder were made using a pitot tube of inner diameter of $0.5 \mathrm{~mm}$. For boundary layer measurements, a pitot tube of 
elliptical cross section with a diameter of $3 \mathrm{~mm}$ was used. The pitot tubes were calibrated in a rectangular air duct at a position where fully developed flow was achieved. The pressures sensed were communicated to a transducer (accuracy $\pm 0.5 \%$ ) associated with a dynamometer unit. The room temperature was constant for most the test time. The reading pressures were uniform within $\pm 0.5 \mathrm{~mm} \mathrm{H}_{2} \mathrm{O}$. The drag coefficient was determined by integrating the measured pressure distributions.

\section{Results and Discussion}

\section{Pressure coefficient}

Fig. 4 shows the time averaged surface pressure distributions around the cylinder of different groove geometries. In this figure, $\theta$ represents the angle measured from the stagnation point. The main behavior of pressure distributions is nearly the same for the smooth and grooved cylinders. Whereas, the location of the minimum pressure coefficient is changed with the groove geometry. The minimum pressure coefficient is located around $\theta=90^{\circ}$ and $270^{\circ}$ for the smooth cylinder. This value is shifted toward the stagnation point when the grooved cylinder Cyl was used. For the other two cylinders (Cy2 and $\mathrm{Cy} 3$ ), the minimum value of the pressure coefficient is shifted toward the base point. The reason can be explained as follows: the grooves behave as large scale and continuous vortex generators. The circulations which were created inside the grooves cannot close inside the grooves and the vorticity lines will go outside as horseshoe vortices. These horseshoe vortices are the main cause for the transition of the laminar boundary layer to the turbulent boundary layer. This means that the change in the groove geometry leads to a change in the horseshoe vortices behavior which affects the pressure field as well as the separation points. These results are in good agreement with that of cavity flows, Achenbach and Heinecke, (1981). The corresponding drag is also affected by this variation. Waka and Yoshino (1987) have investigated the flow around a circular cylinder with variation of the velocity and position of tangential blowing from a surface slot. Based on the measurements of the surface pressure distributions, they demonstrated that a large reduction in the time averaged drag is due to the displacements of the separation points. Therefore, from the pressure distribution shown in Fig.4., it can be concluded that the mechanism of the flow control by means of the present method is similar to that of the tangential blowing from a surface slot.

The pressure coefficient at the base of the cylinder is given in Fig. 5. It is seen that the base pressure coefficient is also affected by the groove shape resulting a change in a flow behavior in the wake region 
as well as the total drag: The base pressure coefficient for $\mathrm{Cy} 3$ is greater than that of other cylinder. For all tested cylinders, the data indicate a linear decrease in $\mathrm{Cp}_{\mathrm{b}}$ with increasing the Reynolds number.

The data suggest the presence of a relationship between the groove geometry and the strength of interaction between the flow in the grooves and the wake flow.

\section{Drag coefficient}

The drag coefficients at different shape geometries of the grooves are shown in Fig.6. Similar to the case of smooth cylinders (Achenbach and Heinecke, 1981) and of other V and square grooved cylinders ( Ko et al. 1987), the distinct regimes of subcritical, critical and supercritical can be identified. However, there is a shift of the flow regime to lower Reynolds numbers. The critical Reynolds number for the flow around a smooth cylinder as given by Achenbach (1968) is $\operatorname{Re}_{\mathrm{cr}} \simeq 3 \times 10^{5}$. However it is clearly observed that the critical Reynolds number of tested grooved cylinder is $\operatorname{Re}_{\text {or }} \simeq 5 \times 10^{4}$.

\section{Velocity distribution}

The distributions of the mean velocity at $\operatorname{Re}=6.85 \times 10^{4}$ are given in Fig.7, for smooth and grooved cylinders at different downstream locations $(X / D=0.2,0.4$ and 0.6 ) from the cylinder. The main behavior of the velocity distributions is almost the same for all tested cylinders. The mean velocity decreases to be minimum near the center of the cylinder. The area of minimum velocity becomes short as one goes from the cylinder downstream and is affected strongly by the grooved shape. This indicates that the flow field in the wake of the cylinder is influenced by the horseshoe vortices created inside the grooves. Therefore, the growth of the boundary layer will be affected by the nature of the horseshoe vortices which indicates instability of the flow ( Roshotko, 1976 ) and this type of flow behaviors is usually found in transition and separating flows.

\section{Boundary Layer Characteristics}

Available data suggest significant differences in the development of the boundary layer associated with flows over smooth, rough and grooved cylinders, Leung and Ko (1987). Therefore, in the present work detailed boundary layer measurement were carried out to investigate the effect of groove shapes. 
In fact, it is commonly known that for the flow over a rough surface, a shift of the origin of the boundary layer velocity profile is necessary in order to satisfy the universal law of the wall, Perry et al. (1969) and Bandyopadyay (1987). Leung and Ko (1987) showed that the same phenomenon occurs when the flows comes over a grooved cylinder and they developed mathematical models to obtain the effective origin of the laminar and turbulent boundary layers.

Leung and Ko (1987) started the model as given by Schlichting (1979) as follows: The displacement and momentum thicknesses of the laminar boundary layer can be expressed by

$\frac{\delta^{*}}{\delta}=\frac{3}{10}-\frac{\mathrm{A}}{120}$

and

$\frac{\delta^{* *}}{\delta}=\frac{1}{63}\left[\frac{37}{5}-\frac{\mathrm{A}}{15}-\frac{\mathrm{A}^{2}}{144}\right]$

Where $\mathrm{A}$ is a nondimensional pressure gradient parameter related to the boundary layer thickness and the boundary layer edge velocity $\mathrm{U}_{\mathrm{E}}$, and is given by

$\mathrm{A}=\delta^{2} / \nu \frac{\mathrm{dU}_{\mathrm{E}}}{\mathrm{dx}}$

Assuming a constant pressure across the whole boundary layer then the velocity gradient can be obtained from the circumferential pressure distribution together with the Bernoulli's Equation. Also, assuming that the vitual origin is located at a vertical displacement from the bottom of the groove. Then $\mathrm{A}, \delta^{*}$ and $\delta^{* *}$ can be evaluated. These values are then compared with those of the measured values until agreement between them is found at a particular $\Delta h$. An unique value of $\Delta \mathrm{h}$ is thus obtained in each case. The results show that the value of $\Delta \mathrm{h}$ differs from laminar to turbulent flow regime as follows:

For laminar boundary layer

$$
\Delta \mathrm{h} / \mathrm{h}=0.0043 \theta+0.43
$$

It has to note that the virtual origin is defined as an origin for the profiles to have the logarithmic distribution of velocity near the wall and can be considered as a measured of the interaction between the mean flow and the roughness (grooves), Bondyopadhyay (1987).

The correct value of $\Delta h$ will be used to calculate the value of $A$ and then $\delta^{*}$ and $\delta^{* *}$. The results will be given latter. 
For the turbulent boundary layer, a method based on the law of wake is employed. According to Coles ( 1956 ), the mean velocity profile can be given by :

$$
\frac{\mathrm{u}}{U_{*}}=\mathrm{F}\left(\frac{\mathrm{y} \mathrm{U}_{*}}{v}\right)+(\Pi / \mathrm{k}) \mathrm{w}(\mathrm{y} / \delta)
$$

where

$F^{\prime}\left(\frac{y U_{*}}{v}\right)$ is the law of the wall and $w(y / \delta)$ and $\Pi$ are the wake function and the wake parameter respectively,

$$
w(y / \delta)=\frac{\frac{u}{U_{*}}-\left(\frac{1}{0.4} \ln \frac{\mathrm{y} U_{*}}{v}+5.1\right)}{\frac{\Pi}{0.4}}
$$

and

$$
\frac{\mathrm{U}_{1}}{\mathrm{U}_{*}}=\frac{1}{\mathrm{~K}} \ln \left(\frac{\delta \mathrm{U}_{*}}{v}\right)+\mathrm{C}+\frac{2 \Pi}{\mathrm{K}}
$$

Where $\mathrm{K}$ and $\mathrm{C}$ are the Von Karman constant and the constant in the law of the wall, respectively. Solving Eqs. 4 - 6 the value of $U *$ can be obtained. An iteration process is carried to obtain the correct origin which would give a constant $U *$. As the correct origin is obtained, the value of $\delta^{*}$ and $\delta^{* *}$ and then $\mathrm{H}$ can be calculated. The result shows that the value of $\Delta \mathrm{h}$ for turbulent boundary layer is given by

$$
\Delta \mathrm{h} / \mathrm{h}=0.0361 \theta^{0.72}
$$

Fig. 8 . shows the variation of boundary layer thickness and the shape factor with the circumferential angle $\theta$ for the smooth and grooved cylinders. It is seen that the boundary layer thickness is affected by the grooves. The values of the boundary layer thickness and shape factor are larger for the grooved cylinders than that of the smooth cylinder. The boundary layer thickness decreases with increase in the Reynolds number. This suggests that the development of the boundary layer of the grooved cylinder is quite similar to that of the smooth cylinder. The shape factor of about 2.2 is observed for both the grooved and smooth cylinder up to $\theta$ $=70^{\circ}$, which signifies the existence of a laminar boundary layer. For $\theta>70^{\circ}$, the value of the shape factor is affected by the groove geometry. For higher angles, the large increase in the shape factor indicates the location of separation. The data of Leung and Ko (1987) are given for comparison. It is seen that the trend of the development of boundary layer thickness and shape factor are in good agreement. 


\section{Conclusion}

The main features of the flow over a grooved cylinder are found to be a function of the groove shape and Reynolds number. Different flow regimes, subcritical, critical and supercritical can easily identified from flow measurements. A shift of the flow regime to lower Reynolds number is found when the grooved cylinders are used. The critical Reynolds number for grooved cylinder at the tested conditions is found around $5 \mathrm{x}$ $10^{4}$. The main behavior of pressure distributions around the smooth and grooved cylinders is the same. Whereas, the location of minimum pressure coefficient for grooved cylinders differs from that of smooth cylinders; resulting a change in separating point as well as the value of drag coefficient. Also, the main behavior of the boundary layer development for smooth and grooved cylinders is the same. However, the values of the boundary layer thickness and shape factor are larger compared to those of a smooth cylinder.

\section{References}

1. Achenbach, E., " Distribution of local pressure and skin friction around a circular in cross flow up to $\operatorname{Re}=5 \times 10^{6}$," Journal of Fluid Mech. Vol.34, 1968, pp. 625 - 639.

2. Achenbach,E., "Influence of surface roughness on the cross flow around a circular cylinder," Journal of Fluid Mech., Vol. 49, 1971, pp. 321-335.

3. Achenbach,E. and Heinecke, E., "On vortex shedding from smooth and rough cylinders in the range of Reynolds number $6 \times 10^{3}$ to $5 \mathrm{x}$ 106" Journal of Fluid Mech., Vol. 109, 1981, pp. 239-251.

4. Bandyopadyay, P.R., " Rough wall turbulent boundary layer in the transition regime," Journal of Fluid Mech., Vol.180, 1987, pp. 231 266.

5. Bearman, P. W. and Harvey, J. K., "Golf ball aerodynamics," Aeronautical Quarterly, May 1976, pp. 112-122.

6. Coles, D. " The law of the wakes in the turbulent boundary layer", Journal of Fluid Mech, Vol. 1, 1956, pp.191 - 226.

7. Gregory, U. S. and Farid, H. M., "Effect of surface grooves on base pressure for a blunt trailing edge airfoil," AIAA Journal, Vol. 28, No. 6, 1990, pp. 1133-1135.

8. Kawamura, T.and Kuwahara, K., "Computation of high Reynolds number flow around a circular cylinder with surface roughness, " AIAA paper, No 84-0340, 1984. 
9. Ko, N. W., Leung, Y. C. and Chen, J. J., "Flow past V-groove circular cylinders," AIAA Jornal, Vol. 25, 1987, pp. 806-811.

10. Leung, Y.C. and Ko, N.W., " Investigation of flow over grooved circular cylinders, "Inter. Conf. of Fluid Mech. Beijing, 1987, pp. 578 $-583$.

11. Naumann, A. and Quadflieg, H., " Vortex generation on cylindrical buildings and its simulation in wind tunnel, "IUTAM - IAHR Symposium, Karlsruhe, 1972, pp.731-747.

12. Perry, A.E, Schefield, W.H. and Joubert P.N., " Rough wall turbulent boundary layers “, Journal of Fluid Mech., Vol. 37, 1969, pp. 383 413.

13.Quass, B., Howard, F., Weinstein, L., and Bushnell, D.," Longitudinal grooves for bluff body drag reduction," AIAA Journal, Vol. 19, No. 4, April 1981, pp. 535-537.

14. Roshotko, E. , " Boundary layer stability and transition, An. Rev. of Fluid Mech., No. 8, 1976, pp. 34 - 349.

15. Schlichting, H., "Boundary Layer Theory", McGraw Hill, 7th ed., 1979

16. Waka, R. and Yoshino, F., "The forced reattachment of the separated shear layer of a circular cylinder with tangential blowing, " Transaction of JSME, Vol .53, No 490, 1987, pp. 1704 - 1710.

\section{Nomenclature}

\begin{tabular}{|c|c|c|c|}
\hline $\mathrm{Cd}$ & drag coefficient & $u \max$. & the maximum velocity \\
\hline $\mathrm{Cp}$ & $\begin{array}{l}\text { pressure coefficient } \\
=\left(\mathrm{p}-\mathrm{p}_{\infty}\right) /\left(\rho u_{\infty}^{2} / 2\right)\end{array}$ & $\mathrm{x}, \mathrm{y}, \mathrm{z}$ & $\begin{array}{l}\text { coordinates of the center and } \\
\text { midspan of the circular cylinder }\end{array}$ \\
\hline Cyl & cylinder No. 1 & $\rho$ & density of air \\
\hline Cy2 & cylinder No. 2 & $\infty$ & free stream conditions \\
\hline Cy3 & cylinder No. 3 & $\mathrm{c}_{\mathrm{r}}$ & critical conditions \\
\hline $\mathrm{D}$ & cylinder diameter & $v$ & kinamatic viscosity \\
\hline $\mathrm{h}$ & groove depth & $\delta$ & boundary layer thickness \\
\hline $\mathrm{H}$ & shape factor & $\delta^{*}$ & displacement thickness \\
\hline $\mathrm{P}$ & pressure & $\delta^{* *}$ & momentum thickness \\
\hline $\operatorname{Re}$ & Reynolds number & $\theta$ & angle measured clockwise \\
\hline S.Cy & : smooth cylinder & & from the stagnation point \\
\hline & : flow velocity & & \\
\hline
\end{tabular}



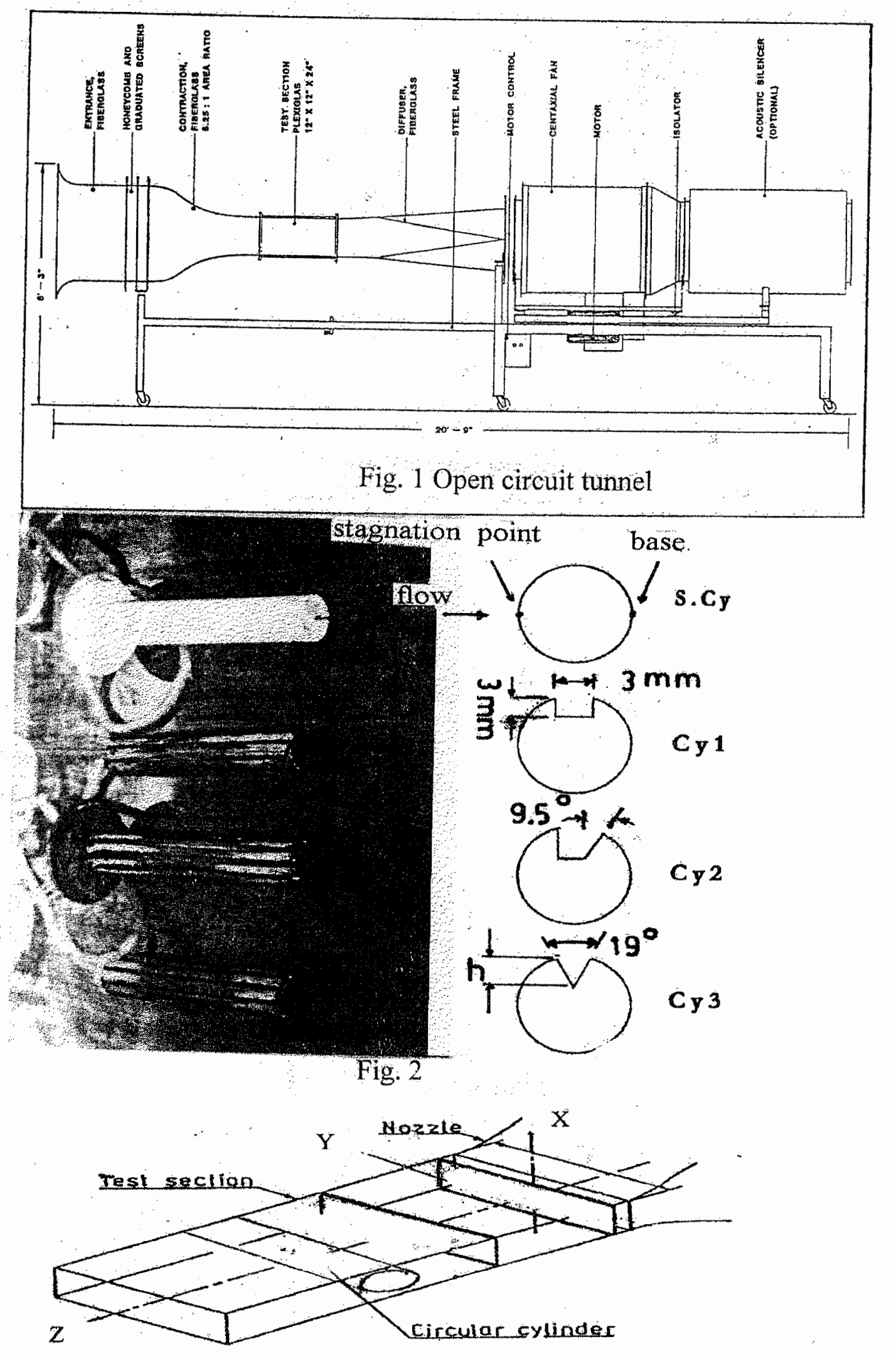

Fig. 3 Coordinate system 

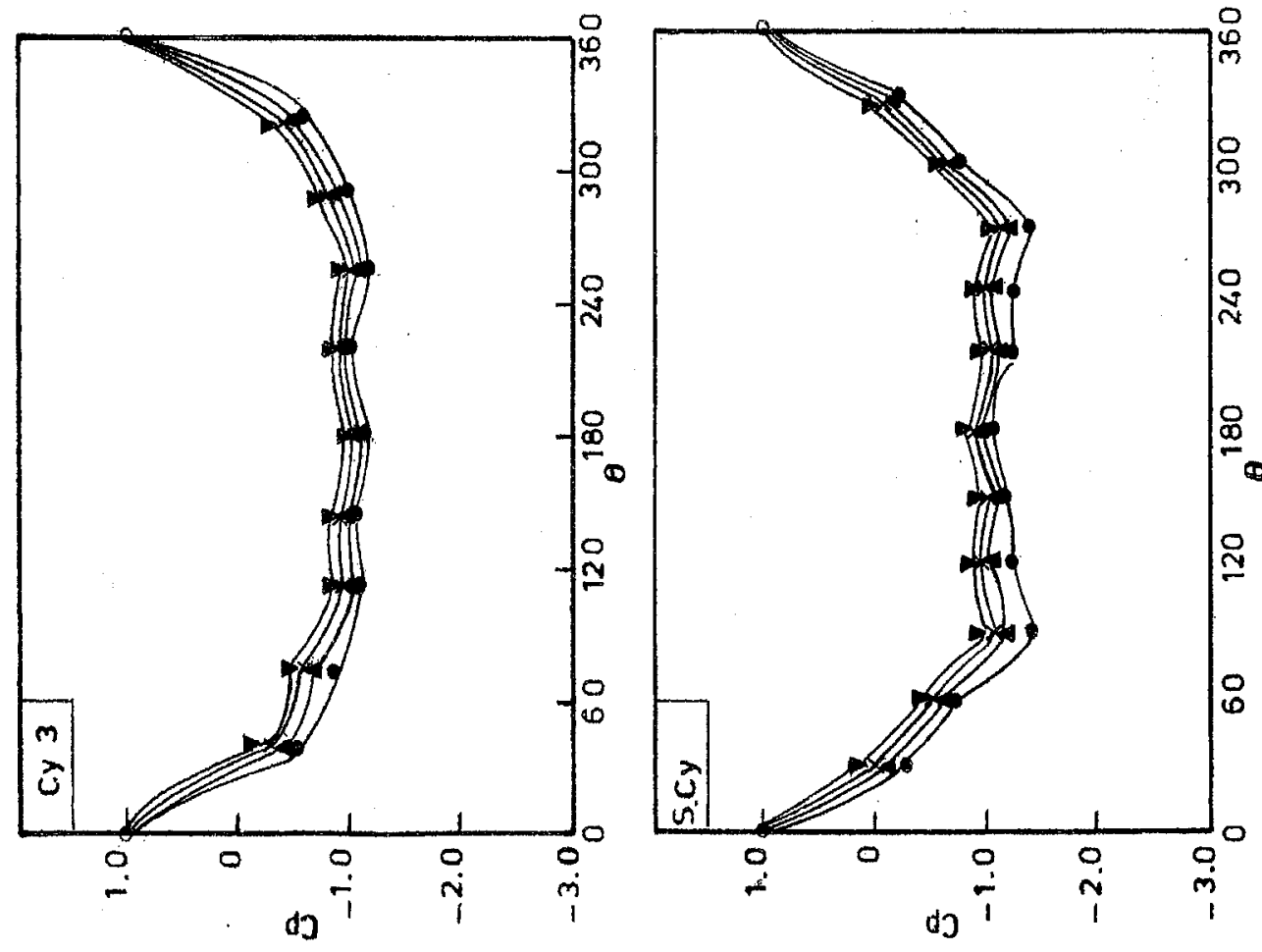

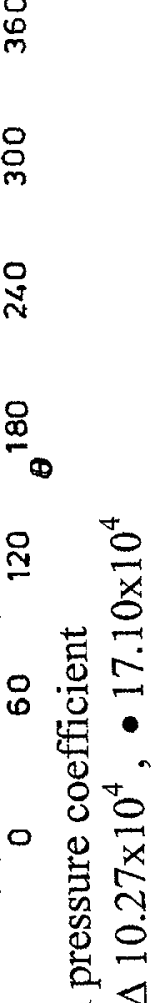

ธ

कृ

3
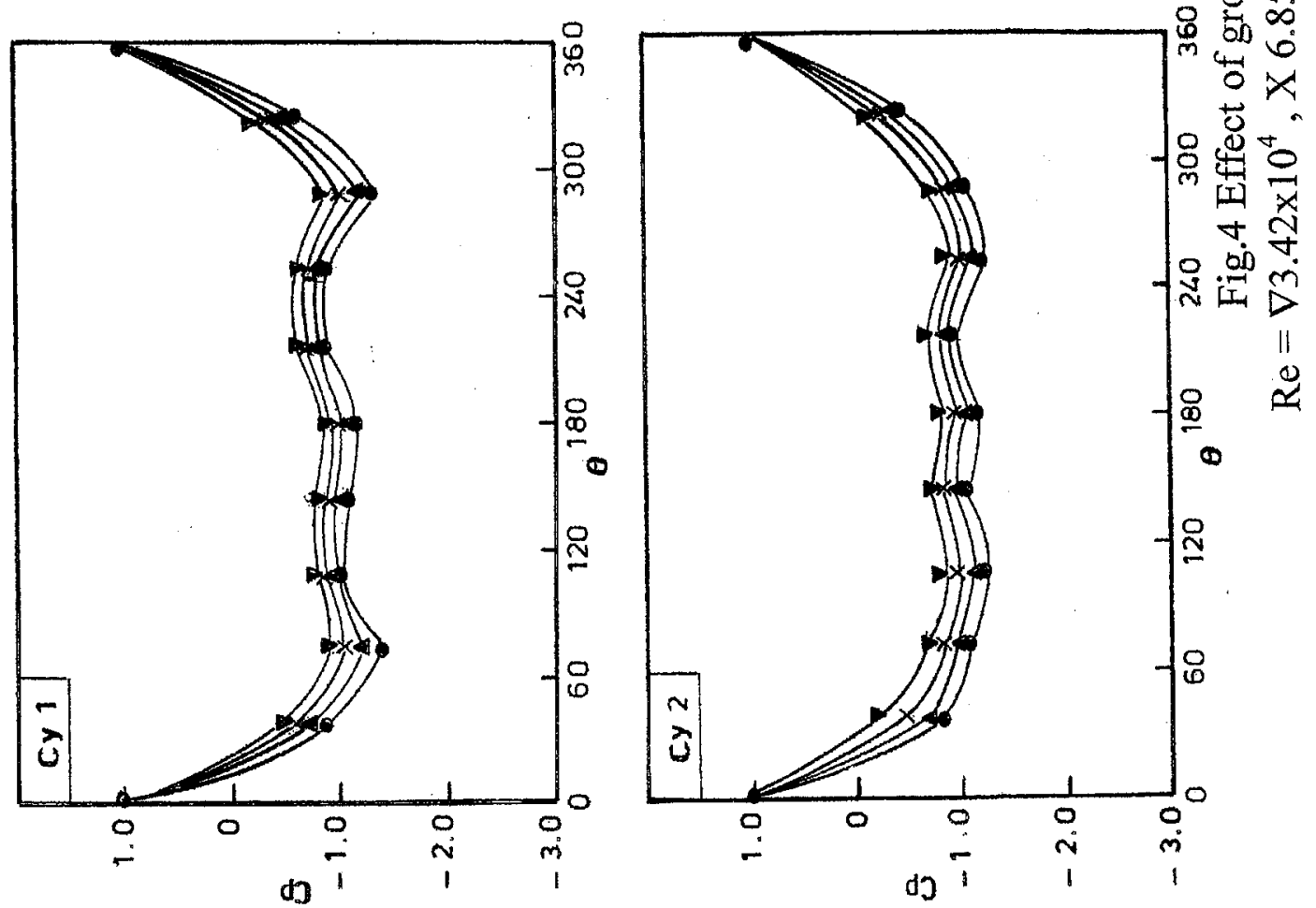


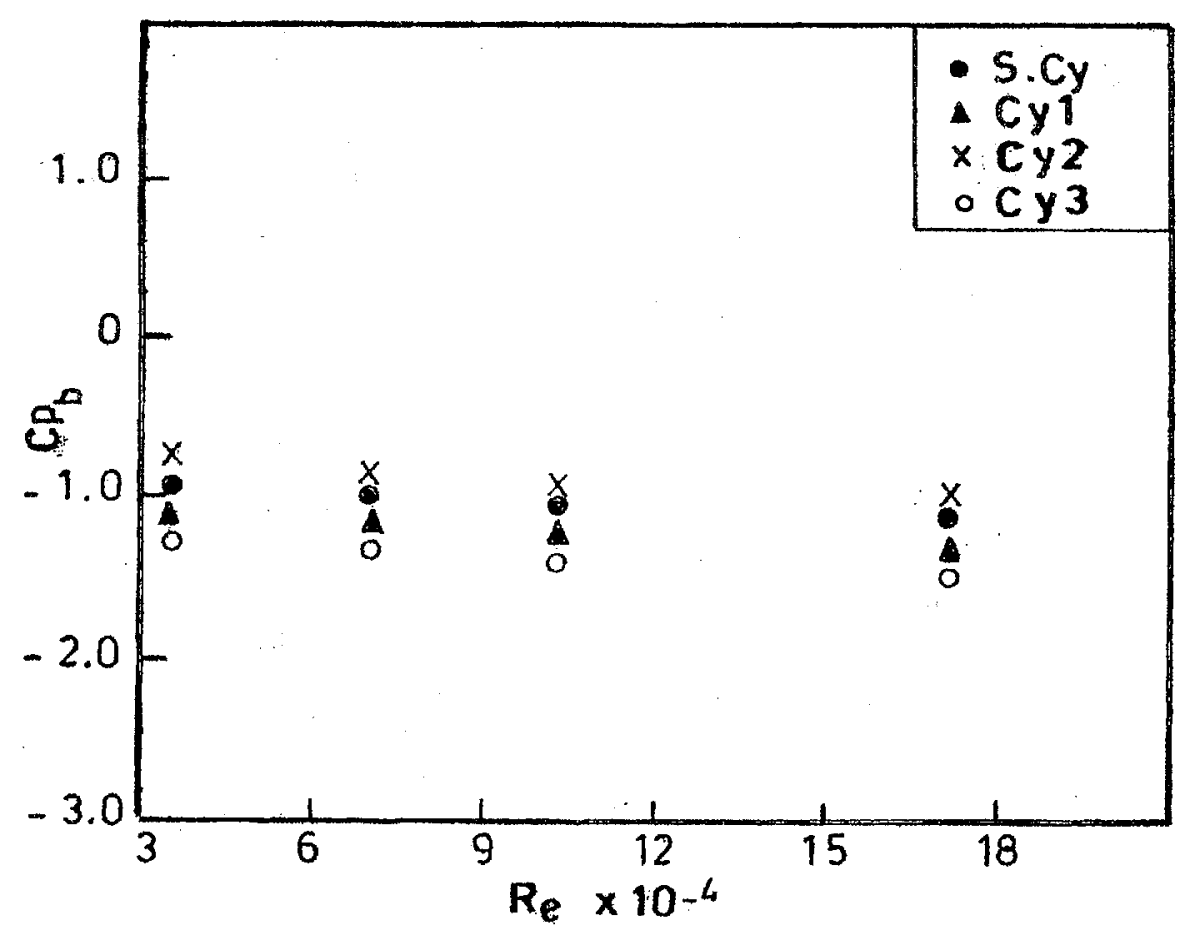

Fig. 5 Variation of the base pressure

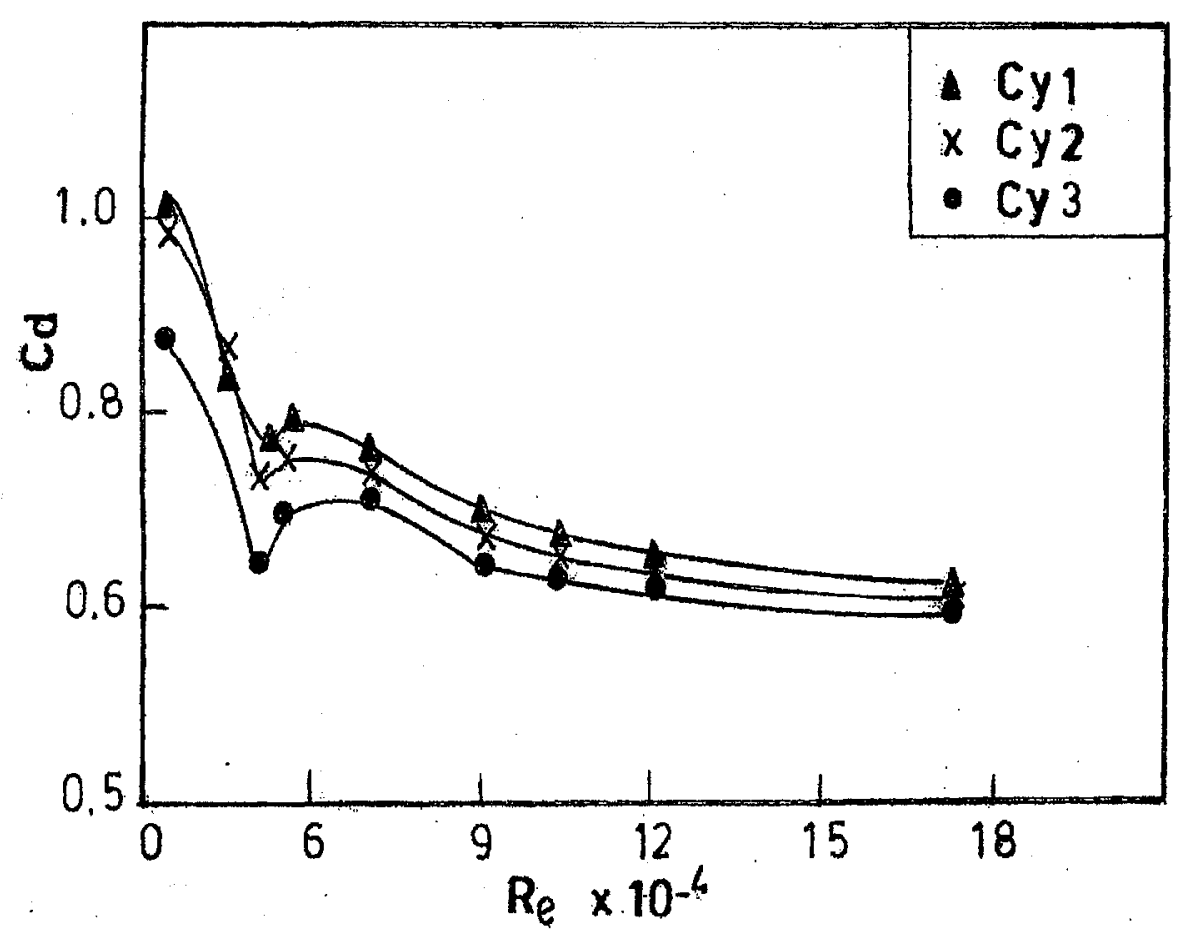

Fig. 6 Drag coefficient for grooved cylinders 

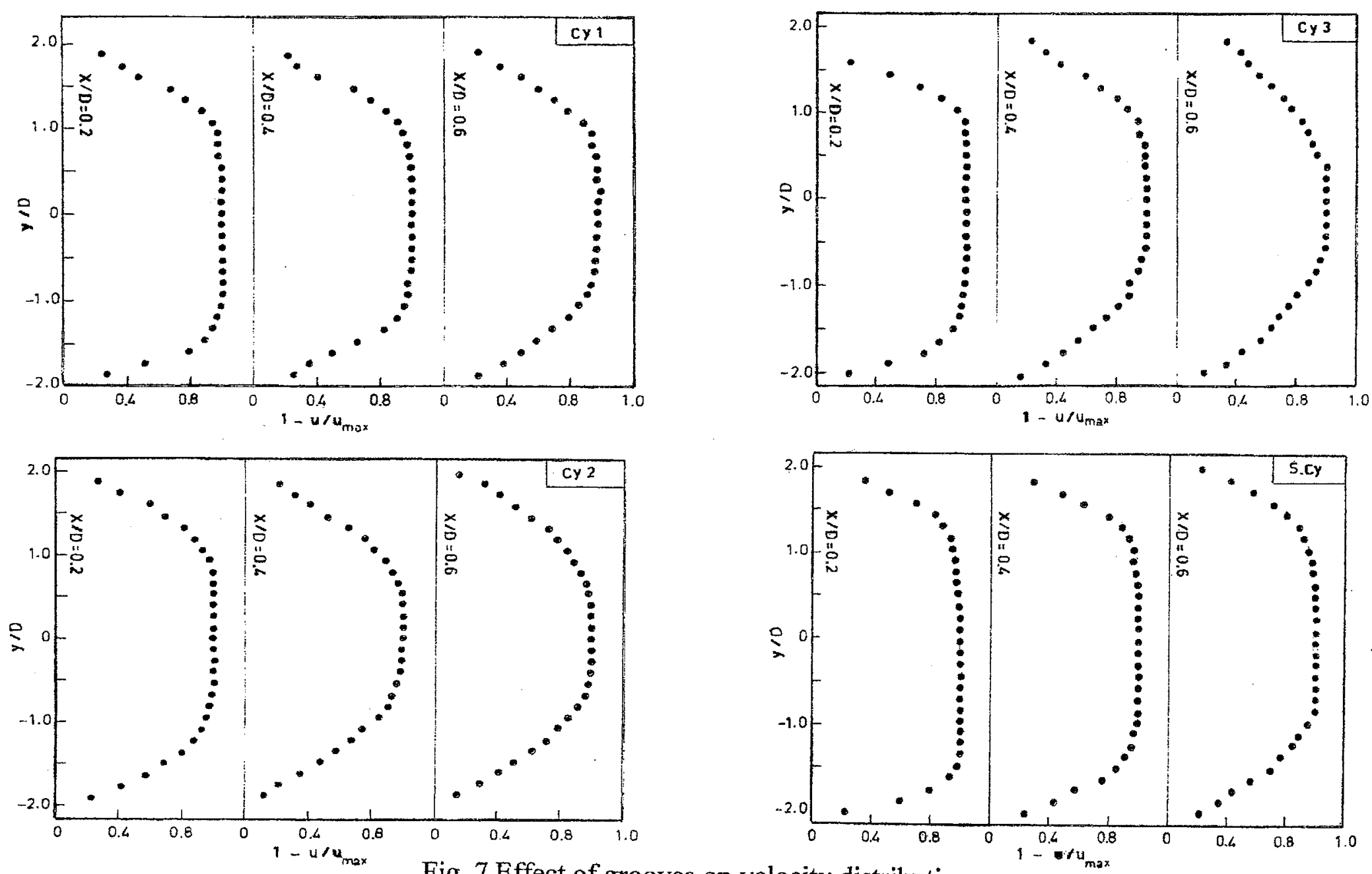

Fig. 7 Effect of grooves on velocity distribution $\operatorname{Re}=6.85 \times 10^{4}$ 

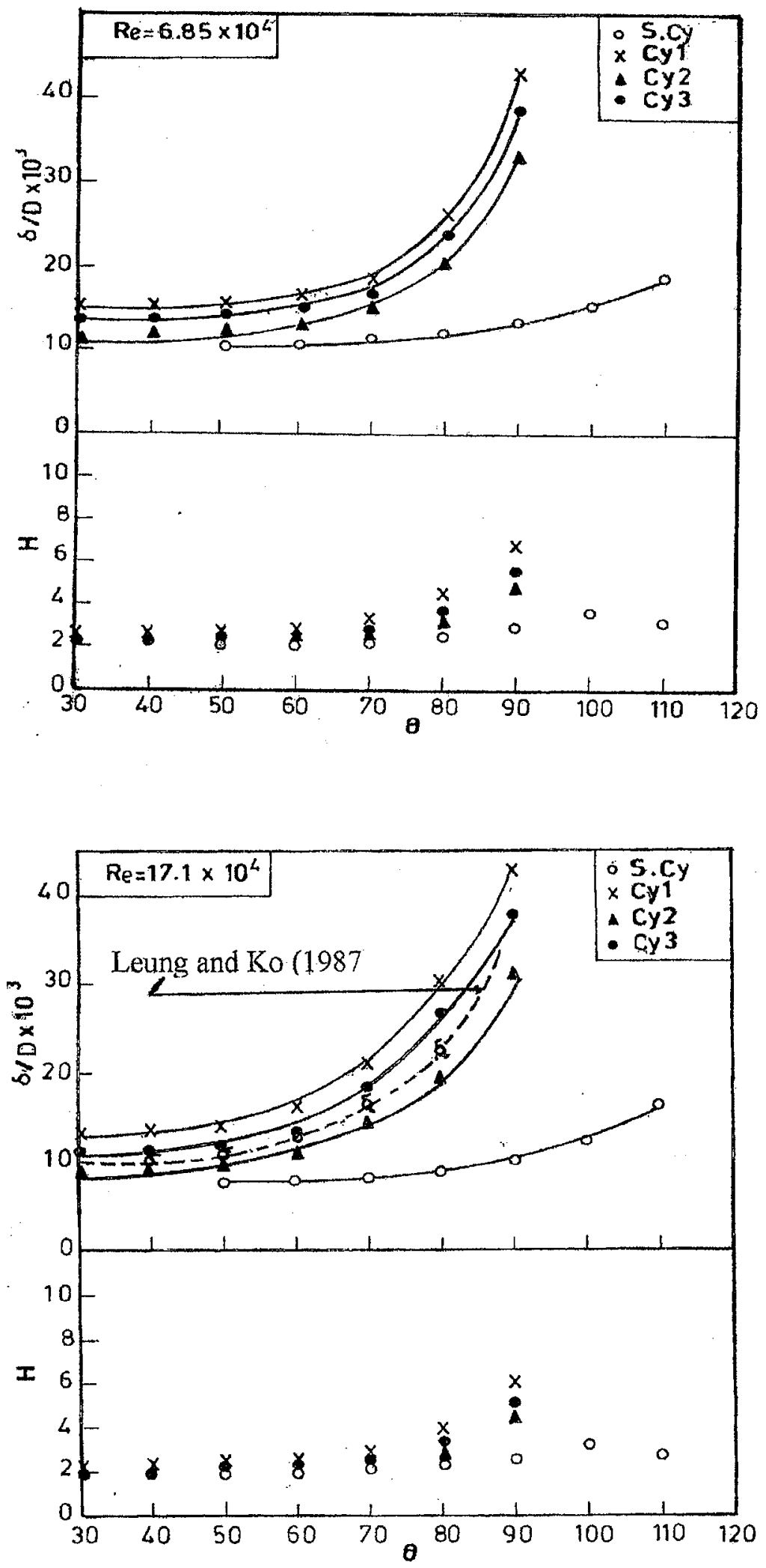

Fig. 8 Effect of grooves on the boundary layer thickness and shape factor 


$$
\text { سريان مائع حول اسطو انة ذات أخدود البحث }
$$

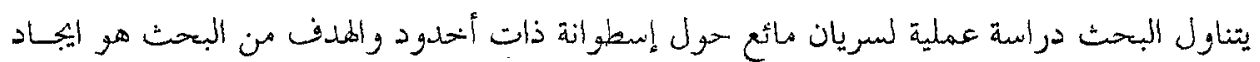

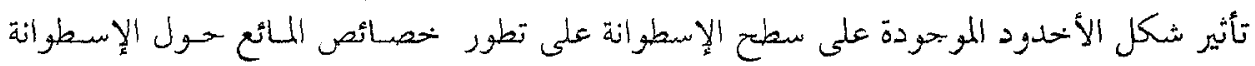

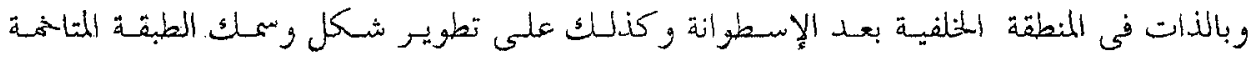

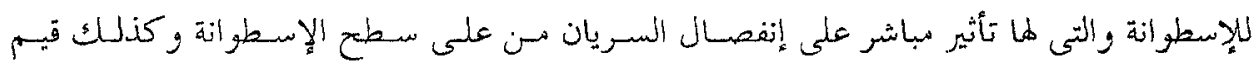

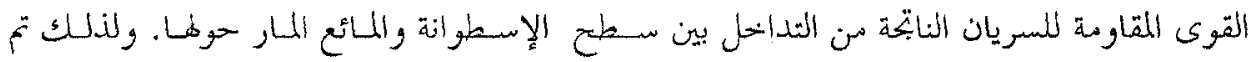

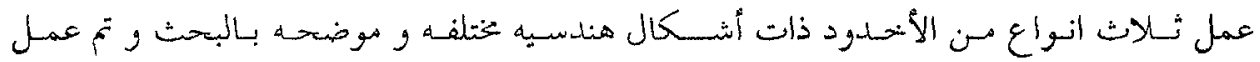
التجارب المعمليه عليها حيث تم قياس توزيع الضغوط حول الإسبطوانه و فى المنطقه الملفيه فلسا و

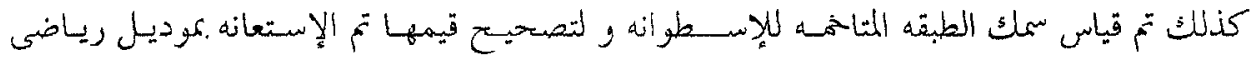

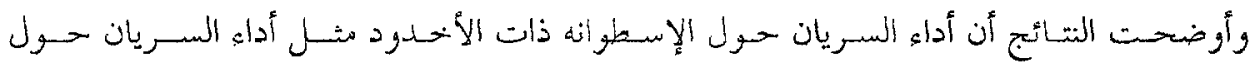

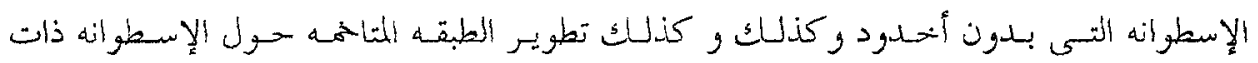

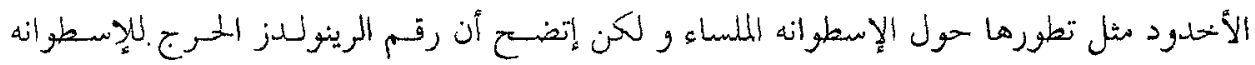

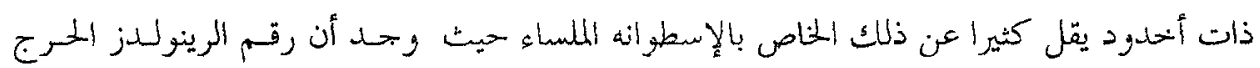

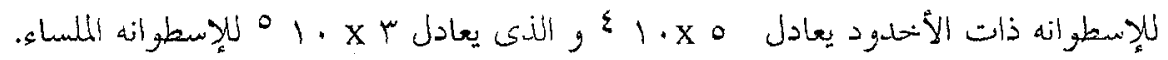

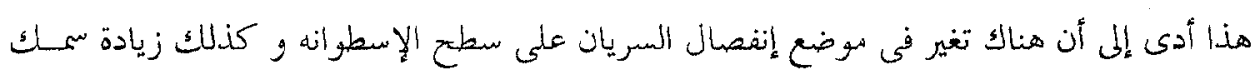

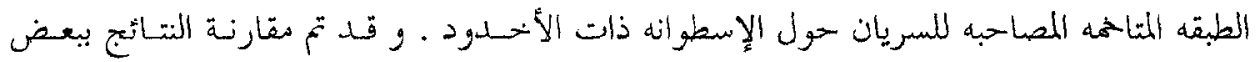
النتائج السابقه المتاحه ووجد تطابق بين الإثنين و يستخدم هذا النوع من السريان في المدانثن ذات العوازرض و الجمسور ذات العوأرض و كذلك كور الجمولف . 